\title{
103
}

\section{Fostering mathematical thinking through gradual progression Computer Assisted Instruction}

\author{
Chen Yun \\ Xiang Juncao \\ Liuzhou Normal School \\ Guangxi \\ China
}

\author{
Su Fanglai \\ The Education Centre \\ Guangxi \\ China
}

\begin{abstract}
Given the basic fact that at present in developing countries Computer Assisted Instruction (CAI) in elementary and secondary classrooms can only be implemented on low grade personal computers, this paper contrasts and analyzes the different functions of computers in teaching. Single or networked personal computer systems can in combination with conventional teaching systems effectively bring the special advantages of CAI into play. Gradual progression through groups of problems makes it possible, even on low grade personal computers, to totally avoid the mechanistic, dumb or even fatal approaches of traditional CAI and helps to fully develop mathematical thinking. The experiences described in this paper show the basics of gradual progression CAI in which the algorithmic and heuristic method are combined to solve progressively more difficult problems in mathematics.
\end{abstract}

Main conference themes: tutoring

Educational areas: primary education

Study topics: mathematics

Secondary keywords: computer assisted instruction, thinking 


\section{INTRODUCTION}

Early traditional Computer Assisted Instruction (CAI) used the microcomputer for procedure based teaching and showed a teaching pattern based on the behavioural model. The educationalists criticized early CIA as: "to impart the same old knowledge by using the little masked same old method" (S. Papert, 1972). "The students face a dumb machine and study unchanging material, and their cerebrum seems to have no room for displaying prowess" (Zhang Dexiu, 1982). As a consequence students become slaves of the microcomputer.

How can this cardinal weakness, the confinement of student thinking, be overcome? The specialists say that artificial intelligence techniques must be introduced and that specialized, computer based multimedia systems must be adopted such as WICAT which can provide study materials in the form of audio, writing, charts and animated cartoons (D. H. Houston, 1985). However, due to the level of economic development in developing countries such as China for a long time to come middle and primary schools will only have access to low grade personal computers. Cultural background and educational system only allow instruction in classes of different grades unifying the teaching plan and the learning progress.

Can CAI in China therefore only exist in the Skinner age? Can traditional CAI only be avoided by adopting specialized multimedia systems? Can students be freed from the dumb instruction procedures of CAI in a personal computer hardware environment? Since 1978 the authors of this paper have been doing research work related to mathematics learning in primary school using low grade microcomputers of the types: LASER310 (CPU: Z80) or CEC-1 (CPU: 6502). This paper discusses some of the knowledge which has been gradually gained in practice.

\section{The microcomputer and information transmission in teaching}

Teaching is realized by transmission and processing of information. Consequently a teaching system is system for transmission and processing of information. The transmission process can be modelled by the transmission pattern diagram proposed by Westly, as shown below.

In the information transmission process of teaching the microcomputer, as a teacher, may play several roles: either as A (transmitter) or C (checking on the way of transmission) or both A and C simultaneously. As a 'checker' it not only controls itself, but also other 'transmitters' excluding the teacher. 


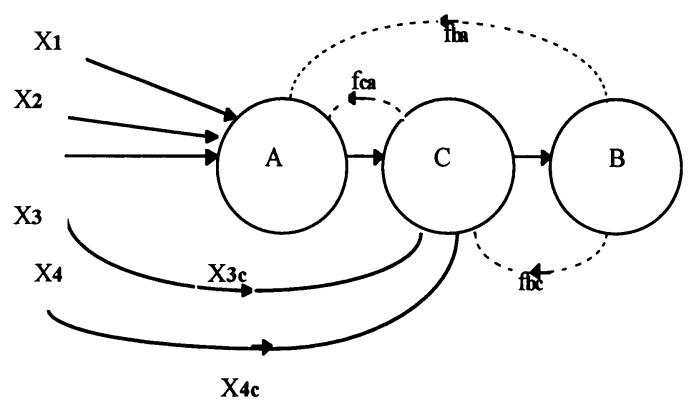

Explanation:

$\mathrm{X}$ : environmental information;

A: source of information, i.e. information transmitter;

C: checks the way of transmission, B: information receiver; fba: feedback from receiver to transmitter;

fbc: feedback from receiver to checker; fca: feedback from checker to transmitter.

Fig. 1 The Westly transmission pattern diagram

The modern information transmission system of teaching may be divided into the following different forms according to the different roles of the microcomputer:

System 1: The microcomputer does not participate in the transmission; or only participates as a 'transmitter'. In this case the 'checker' is a teacher.

System 2: Only the microcomputer together with the 'receiver' constitute a transmission system in which the microcomputer is a 'transmitter' and a 'checker' as well.

System 3: The microcomputer together with other 'transmitters', excluding the teacher, form a transmission system in which the microcomputer is a 'checker'.

Of the systems mentioned System 1 is a so-called 'conventional instruction system' in which the microcomputer which serves as a source of information does not change its identity. The fundamental defect of this system is that a teacher who is dealing with many students, is forced to adopt a single speed and a straight line type of instruction procedure. When the microcomputer is introduced as a checker, the students may then study various content at different speeds which effectively remedies the defect in System 1.

System 3 is a so-called specialized system of CAI. Although delivering the instruction on certain content all on its own, it cannot replace the teacher completely. Consequently even if this 'specialized' system is available, teaching still has to use System 1. In other words, teaching is done through a 
combination of the Systems 1 and 3 (identified by ' $1+3$ ' for short). However, at present education in middle-primary schools in developing countries only uses combinations of Systems 1 and 2 (' $1+2$ ' for short). And as a consequence teaching relies more on System 1. However, whether or not CAI follows a traditional, mechanical or dumb pattern mainly depends on the design of the course components and not so much on the type of system.

\section{The material and phase of study}

In practice the pupils' learning process with respect to mathematics implies transmission of information from the study materials into the brains of students to produce a change in their thinking. In teaching the carriers of information are mainly characters, symbols, diagrams, tables, curves and other teaching aids; its means of delivery are mainly mechanical waves (sound) and electromagnetic waves (light, electricity) [2]. According to the form of carrier we may roughly divide the study materials into two kinds: image and language. After the study materials have been read into the cerebrum, these are transformed into internal image or internal language. The thinking which is performed with the aid of these two thinking tools, can be identified as image thinking and abstract thinking.

A simple personal computer may be clumsy in producing sound and image. Therefore System 2 mainly uses the screen to provide language based study material to the students. However all other study materials in System 3 can also be provided by System 1. Therefore the distinction between the ' $1+2$ ' and ' $1+3$ ' forms of teaching lies only in the fact that individualization is brought into more full and thorough play in the latter.

In a complete study process the student must not only read the study material provided by the 'transmitter' into his cerebrum to trigger thinking (internal transformation), but students must also pass the results of their thinking in the form of certain materials back to the 'transmitter' in order to acquire KR (Knowledge Result) information. The results of the thinking process are transformed into output (external transformation). The internal transformation and the external transformation of the thinking process with respect to mathematics may be divided into some stages. In the internal transformation the student may focus his main attention on understanding and thinking, and output some simple information; in the phase of external transformation the acquired primary knowledge is expressed in a more complete form. The newly created structure of cognition is consolidated further in a process of frequent and complicated information exchange with the outside world. When teaching in ' $1+2$ ' mode we may let the students internally transform the study material in System 2 (thinking) and work related to external transformation and arranging may continue in System 1. An individual approach 
is much more important in the phases of internal transformation and thinking than in the phases of external transformation and arrangement. Here the instruction pattern ' $1+2$ ' is very effective.

\section{The instruction method of algorithm and heuristics}

Both the algorithmic and the heuristics approach were proposed by L. de Leeuw (1975) on the basis of his research on teaching students how to think by means of the microcomputer. The algorithmic approach teaches students to complete all thinking steps following a constant pattern and a fixed line of thought. The heuristics approach lets students try their best to find and execute the steps for the solving of the problem; in other words, when the students are unable to solve the problem, they are pointed in the right way by giving stronger and stronger clues to lead them to the answer. De Leeuw has argued that the heuristics approach may produce a better effect with more complicated problems. However, for limited or similar problems the algorithmic approach may be best.

If in primary school mathematics we define 'teaching thinking' as making students learn to solve a certain type of problem, then the algorithmic approach is certainly effective. But, if we define 'teaching thinking' as making pupils not only able to 'do so', but also able to know 'why it is done so', then the instruction method of heuristics is necessary.

With respect to problem solving G. Polya had pointed out: "Have you ever seen it before? ... Do you know a problem related to it? ... Here is a problem which is relevant to your current problem and has been solved long ago. Can you use it? ... Can you solve part of this problem". These words tell us that an important way to find out how to solve problems is to transfer the problem into one or more known problems. In other words 'clues' obtained in heuristics may be a group of 'solved problems' or 'problems easier to solve' which progressively close in on the present problem. This is what is meant by a 'progressive group of problems' in the following paragraphs.

When faced by a 'progressive group of problems' pupils can only solve a problem with their own experience and mode of thinking which both avoids stereotyped dullness and imposing the mode of thinking. Because pupils have to identify patterns and find tactics by themselves no unwieldy expert system has to be created. By nature this heuristics approach can be supported by a low grade personal computer.

Through the heuristics approach algorithms obtained by the pupil may have nothing in common and may perhaps be not standard and succinct; therefore a 'group of coordinate problems' is needed for comparing various algorithms, so that pupils can select standard and succinct algorithms from these. After this 
has been done the pupils will also be trained by the algorithmic teaching method to master these algorithms.

\section{CAI with 'gradual progression through a group of problems'}

Without problems there mathematics would not exist and not have been developed. The history of mathematics is one of continuously finding and solving problems. The process of mathematics learning is like a move from individuality to 'classification' may always be described as finding problems and solving problems.

As in other significant areas of learning there are three kinds of mathematical learning methods: representative learning, concept learning and assigned topic learning (Shao Ruizhen et al. 1982). These methods (representative, concept and assigned topic learning) can be expressed in the affirmative or negative form and may be transformed into problems expressed in the interrogative form. The abstract and general problem may always be transformed and resolved into concrete and individual problems. In this way the process of mathematics learning takes the form of solution of a group of problems. For example 'the method for reading multidigit numbers' may be transferred into a series of problems such as 'how is this multidigit number read', 'how is that multidigit number read', 'how are these multidigit numbers read, ... up to 'what is the rule to read the multidigit number', etc. Once these problems have been solved the reading method for the multidigit number is mastered.

Learning may be described in an internal model of the learner: the computer can be used not only to simulate the environment, but also to 'play the part of learners' (W. Bewley et al. 1975). When learning takes place in the form of problem solving, pupils may not only guess, try out and observe the response of the computer environment, but also demand a demonstration from the computer; then they can identify themselves with the action of 'other learner' in the simulation. Following the theory of learning formation of Olson and Bruner (1974) the above mentioned methods of mathematics learning can to a certain extent be considered as directly experienced or observational. Obviously it is more easily accepted by pupils than the learning of a 'symbolic system'.

In general reviewing and introducing new teaching content, consolidation and application, etc. are all included in the process of mathematics teaching in primary school. Using low grade personal computers in CAI these activities can be carried out through the solving of a group of problems. A teaching link may consist of some groups of problems with a progressive deepening of the teaching. At the same time the relationship between groups of problems is also progressive from one to the other. This is the basic structure of gradual progression CAI, moving through a progressive group of problems. 


\section{CONCLUSION}

In this paper ways are described for effective CAI in a hardware environment of low grade personal computers in primary school and middle schools in developing countries. Our first conclusion is that the personal computer system combined with the conventional teaching system may partly solve the problem of individualization in teaching. Here, CAI has the chief task of stimulating internal transformation of the learning material so as to form thinking; the remaining tasks are undertaken by conventional teaching. The second conclusion is that with CAI the learning process of mathematics can be transformed into that of problem and solution; the teaching procedure can take the form of a progressive group of problems. As a learning environment the computer can let pupils learn by 'direct experience'. At the same time the computer can act as 'another learner' and let pupils learn 'observationally'. Our third conclusion is that CAI for mathematics in the primary school should apply both a heuristics and an algorithmic approach. Heuristics is adopted to guide students in summing up their own problem solving experiences, and in thinking out their own possible answers. The algorithmic approach is adopted to train students to master standard, simple algorithms. In this way it is possible to free students from mechanical, dumb and traditional CAI.

\section{REFERENCES}

1. Fu Derong (1990) Computer and Education. The Press of Huazhong Teachers' University.

2. Wan Jiaruo et al. (1991) The Technology in Modern Education. The Technical Press of Science Academy of China.

3. Wan Jiaruo (1990) Computer Assisted Instruction. The Press of Science Academy of China. 